\title{
RNA interference-mediated knockdown of translationally controlled tumor protein induces apoptosis, and inhibits growth and invasion in glioma cells
}

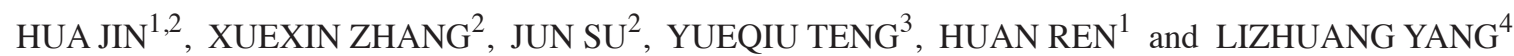

\begin{abstract}
${ }^{1}$ Department of Immunology, Harbin Medical University, Harbin, Heilongjiang 150081; ${ }^{2}$ Department of Neurosurgery, The Third Affiliated Hospital of Harbin Medical University, Harbin, Heilongjiang 150040; ${ }^{3}$ Stem Cell Research Institute, The First Affiliated Hospital of Harbin Medical University, Harbin, Heilongjiang $150001 ;{ }^{4}$ Department of Neurosurgery, The Second Affiliated Hospital of Harbin Medical University, Harbin, Heilongjiang 150081, P.R. China
\end{abstract}

Received September 30, 2014; Accepted June 11, 2015

DOI: $10.3892 / \mathrm{mmr} .2015 .4280$

\begin{abstract}
Translationally controlled tumor protein (TCTP) is a highly conserved, growth-associated and small molecule protein, which is highly expressed in various types of tumor cell. TCTP can promote the growth and suppress apoptosis of tumor cels. However, few studies have reported the effects of TCTP in gliomas. In the present study, a glioma cell line was established, which was stably transfected with TCTP short hairpin ribonucleic acid (shRNA), to investigate the impact of downregulated expression of TCTP on the proliferation, apoptosis and invasion of glioma cells. Western blot and reverse transcription-quantitative polymerase chain reaction analyses demonstrated that TCTP shRNA effectively reduced the expression of TCTP in the U251 glioma cell line. MTT and colony formation assays revealed that downregulated expression of TCTP significantly inhibited glioma cell proliferation. Cell cycle analysis using flow cytometry revealed that the cells in the pRNA-H1.1-TCTP group were arrested in the G0/G1 phase of the cell cycle. Western blot analysis detected downregulated expression levels of cyclins, including Cyclin D1, Cyclin E and Cyclin B. Annexin V-fluorescein isothiocyanate/propidium iodide and Hoechst staining demonstrated that the apoptotic rate of the cells in the pRNA-H1.1-TCTP group was significantly higher than that of the cells in the pRNA-H1.1-control group, with upregulated expression levels
\end{abstract}

Correspondence to: Dr Huan Ren, Department of Immunology, Harbin Medical University, 157 Baojian Road, Harbin, Heilongjiang 150081, P.R. China

E-mail: huanren0102@163.com

Dr Lizhuang Yang, Department of Neurosurgery, The Second Affiliated Hospital of Harbin Medical University, 246 Xuefu Road, Harbin, Heilongjiang 150081, P.R. China

E-mail: lizhuangyang@126.com

Key words: translationally controlled tumor protein, glioma, short hairpin RNA, invasion, apoptosis, gene therapy of B-cell-associated X protein and cleaved-caspase-3 and downregulated expression of B-cell lmyphoma-2 in the apoptotic process. Wound healing and Transwell assays revealed that downregulated expression of TCTP significantly inhibited the migration and invasiveness of the glioma cells; and the expression levels and activities of matrix metalloproteinase (MMP)-2 and MMP-9 were also significantly affected. In conclusion, the present study demonstrated that downregulated expression of TCTP significantly inhibited proliferation and invasion, and induced apoptosis in the glioma cells. These results suggested that TCTP may be important in glioma development and metastasis. Therefore, TCTP is expected to become an effective target for glioma gene therapy.

\section{Introduction}

Glioma is the most common type of malignancy in the central nervous system (1), accounting for more than half of all intracranial tumors, with an incidence rate of 6.5/100,000 individuals (2) and an average survival duration of 14 months (3). The five-year survival rate of malignant glioma is <5\% (4). Glioma is characterized by rapid growth, high levels of invasiveness, frequent postoperative recurrence and high mortality rates. Although the range of comprehensive treatments for glioma, including surgical resection, radiotherapy and chemotherapy, have been improving, the therapeutic effect remains inadequate $(5,6)$. Therefore, clarifying the mechanisms of glioma invasion and metastasis, and determining effective treatment methods, remains a focus of investigation.

Translationally controlled tumor protein (TCTP) is a highly conserved hydrophilic protein, which was identified in 1989 (7). The expression levels of TCTP in a variety of tumor cells are markedly higher, compared with those in normal tissues (8-11). Previous studies have indicated that TCTP is important in the regulation of cell proliferation, cell cycle, malignant metastasis and anti-apoptotic effects (12-16). Additionally, TCTP is associated with the development and progression of glioma (17). In vitro and in vivo experiments have demonstrated that abnormally high expression levels of TCTP in glioma cells can promote cell proliferation, and 
that this promotion of proliferation can be eliminated by downregulation of the expression of TCTP expression (18). The expression of TCTP is also closely associated with tumor deterioration and the sensitivity of tumor cells to drugs (19). The over-expression of TCTP in cells has been observed to significantly inhibit 5-fluorouracil (5-Fu)-induced apoptosis of ovarian cancer and osteosarcoma cells. Following silencing of the expression of TCTP using an antisense oligonucleotide, the sensitivity of U2OS osteosarcoma cells to 5-Fu is enhanced, and the apoptotic rate is significantly increased $(20,21)$. However, the role of TCTP in the occurrence and development of glioma remains to be fully elucidated and further investigation is required.

In the present study, the expression of TCTP in glioma cells was downregulated using RNAi to investigate its effects on the proliferation, apoptosis, metastasis and invasion of the glioma cells, and to examine the associated mechanisms. This investigation suggested that TCTP may be a potential target for the treatment of glioma.

\section{Materials and methods}

Cell lines. The U251, A172, U87-MG and SHG-44 human glioma cell lines were purchased from the Shanghai Institute of Biological Sciences, Chinese Academy of Sciences (Shanghai, China). The U373 cells were purchased from American Type Culture Collection (ATCC, Manassas, VA, USA). The U251, U373, A172 and U87-MG cells were cultured in Dulbecco's Modified Eagle Medium (DMEM, Gibco Life Technologies, Grand Island, NY, USA) containing 10\% fetal bovine serum (FBS, GE Healthcare, Logan, UT, USA) at $37^{\circ} \mathrm{C}$ and $5 \% \mathrm{CO}_{2}$. The SHG-44 cells were cultured in RPMI-1640 medium (Gibco Life Technologies) containing 15\% FBS (GE Healthcare) at $37^{\circ} \mathrm{C}$ and $5 \% \mathrm{CO}_{2}$.

Construction of a TCTP short hairpin (sh)RNA eukaryotic expression plasmid and screening for a stably transfected cell line. The TCTP interfering sequences were designed, according to the TCTP mRNA sequence in GenBank using shRNA designing software, as shown in Table I. The obtained interfering sequences (Wanleibio, Shenyang, China) were ligated into the pRNA-H1.1 eukaryotic expression vector (GenScript, Nanjing, China) using the restriction sites of HindIII and BamHI, and the resulting plasmid was termed pRNA-H1.1-TCTP. U251 cells in the logarithmic growth phase were seeded into 6-well plates. pRNA-H1.1-TCTP was transfected into the U251 cells using Lipofectamine 2000, according to the manufacturer's instructions (Invitrogen Life Technologies, Carlsbad, CA, USA). After 24 h, complete DMEM, containing $400 \mu \mathrm{g} / \mathrm{ml}$ G418 (Invitrogen Life Technologies) was added into each well for screening for 7-14 days, and the clones exhibiting positive expression of TCTP were selected and identified by reverse transcriptionquantitative polymerase chain reaction (RT-qPCR) and western blotting. In the process of the experiment, an untransfected group (parental) and an empty vector-transfected group (pRNA-H1.1-control) were set up as the controls.

Western blot analysis. Following lysis of the cells using NP-40 lysis buffer (Beyotime Institute of Biotechnology,
Shanghai, China), the total cellular proteins were extracted, and the protein concentrations were determined using a Bicinchoninic Acid Protein Assay kit (Beyotime Institute of Biotechnology). Equal quantities of proteins $(40 \mu \mathrm{g})$ were subjected to 10 or $12 \%$ SDS-PAGE, and the proteins were then transferred onto a polyvinylidene difluoride membrane (EMD Millipore, Bedford, MA, USA), followed by incubation overnight at $37^{\circ} \mathrm{C}$ with rabbit anti-human poly clonal antibodies against TCTP antibody (1:200; cat. no. sc-133131; Santa Cruz biotechnology Inc., Santa Cruz, CA, USA), cyclin D1 antibody (1:1,000; cat. no. WL0205), cyclin E antibody (1:1,000; cat. no. WL0055), cyclin B antibody (1:1,000; cat. no. WL0023), Bax polyclonal antibody (1:1,000; cat. no. WL0101), Bcl-2 antibody (1:1,000; cat. no.WL0104), cleaved-caspase-3 antibody (1:1,000; cat. no. WL0146), MMP-2 antibody (1:1,000; cat. no. WL0657), MMP-9 antibody (1:1,000; cat. no. WL0884) or $\beta$-actin antibody $(1: 1,000$; all from Wanleibio, Shenyang, China) at $4^{\circ} \mathrm{C}$. Subsequently, the membrane was incubated with the corresponding horseradish peroxidase-labeled goat anti-rabbit IgG secondary antibody at a 1:5,000 dilution (Beyotime Institute of Biotechnology) for $45 \mathrm{~min}$ at $37^{\circ} \mathrm{C}$. Signals were detected using enhanced chemiluminescence (ECL) solution (Qihai Biotec, Shanghai, China) and the band intensities were normalized against those of $\beta$-actin using Gel-Pro-Analyzer version 4.0 software (Media. Cybernetics, Inc., Bethesda, MD, USA).

$R T-q P C R$. Total cellular RNA was extracted from the cells in each group, strictly according to the instructions of the total RNA extraction kit (Tiangen Biotech, Co., Ltd., Beijing, China). The cDNA $(20 \mu \mathrm{l})$ was obtained by RT using M-MLV Reverse Transcriptase (BioTeke, Beijing, China), and quantitative fluorescence analysis was performed using SYBR Green MasterMix (10 $\mu$; Solarbio, Beijing, China) in an Exicycler $^{\mathrm{TM}} 96$ quantitative fluorescence analyzer (Bioneer, Daejeon, Korea). The concentration of each primer was $10 \mu \mathrm{M}$. The reaction conditions were as follows: $95^{\circ} \mathrm{C}$ for $10 \mathrm{~min} ; 95^{\circ} \mathrm{C}$ for $10 \mathrm{sec}, 60^{\circ} \mathrm{C}$ for $20 \mathrm{sec}$ and $72^{\circ} \mathrm{C}$ for $30 \mathrm{sec}$, for a total of 40 cycles. With $\beta$-actin as the internal control, the $2^{-\Delta \Delta \mathrm{Ct}}$ method (22) was used to analyze the mRNA expression levels of TCTP. The sequences of the primers used are listed in Table II.

MTT assay for cell proliferation. Cells in the logarithmic growth phase were harvested and seeded into 96-well plates at a density of $3 \times 10^{3}$ cells/well. Following culture of the cells for 12, 24, 48, 72 and $96 \mathrm{~h}$, MTT solution (final concentration, $0.2 \mathrm{mg} / \mathrm{ml}$; Sigma-Aldrich, St. Louis, MO, USA) was added into the each well. The cells were continuously cultured at $37^{\circ} \mathrm{C}$ for $4 \mathrm{~h}$, and the supernatant was then discarded. The purple crystals were dissolved using $200 \mu$ l dimethyl sulfoxide (Sigma-Aldrich), and the optical density $\left(\mathrm{OD}_{490}\right)$ values were measured using a microplate reader (ELX-800; Bio-Tek Instruments Inc, Winooski, VT, USA). A cell growth curve was plotted and statistical analysis was performed.

Colony formation assay. The cells in each group were seeded (200 cells/dish) into a $35 \mathrm{~mm}$ petri-dish for culture. Subsequent to the formation of colonies, $4 \%$ paraformaldehyde (Guoyao, Shenyang, China) was used to fix the cells for $20 \mathrm{~min}$, followed 
Table I. shRNA sequences.

\begin{tabular}{ll}
\hline Primer & \multicolumn{1}{c}{ Sequence (5'-3') } \\
\hline $\begin{array}{l}\text { TCTP shRNA } \\
\text { Forward }\end{array}$ & GATCCCCGATGGTCAGTAGGACAGAATTCAAGAGATTCTGTCCTACTGACCATCTTTTT \\
Reverse & AGCTAAAAAGATGGTCAGTAGGACAGAATCTCTTGAATTCTGTCCTACTGACCATCGGG \\
Control shRNA & \\
Forward & GATCCCCTTCTCCGAACGTGTCACGTTTCAAGAGAACGTGACACGTTCGGAGAATTTTT \\
Reverse & AGCTAAAAATTCTCCGAACGTGTCACGTTCTCTTGAAACGTGACACGTTCGGAGAAGGG
\end{tabular}

TCTP, translationally controlled tumor protein; shRNA, short hairpin ribonucleic acid.

Table II. Sequences of the primers used for quantitative polymerase chain reaction.

\begin{tabular}{ll}
\hline Primer & \multicolumn{1}{c}{ Sequence (5'-3') } \\
\hline TCTP & \\
Forward & GCCGTCGTCGTCTCCCTTCA \\
Reverse & ACCCGTCCGCGATCTCCCG \\
$\beta$-actin & \\
Forward & CTTAGTTGCGTTACACCCTTTCTTG \\
Reverse & CTGTCACCTTCACCGTTCCAGTTT \\
\hline
\end{tabular}

TCTP, translationally controlled tumor protein.

by Wright-Giemsa staining (Jiancheng Bioengineering Institute, Nanjing, China) for 5-8 min. The number of colonies, defined as a cell group with $\geq 50$ cells, were counted and recorded under an inverted microscope (AE31; Motic Electric, Xiamen, China). The colony formation rate was then calculated according to the following formula: Colony formation rate $=($ number of colonies $/$ number of seeded cells $) \times 100 \%$.

Detection of the cell cycle using flow cytometry. The cells were cultured in DMEM medium and were harvested on reaching $\sim 90 \%$ confluence. The cell cycle was detected using a flow cytometer (FACSCalibur; BD Biosciences, San Jose, CA, USA) using a Cell Cycle Analysis kit (Beyotime Institute of Biotechnology). In brief, the cells were harvested, washed with phosphate-buffered saline (PBS), and fixed with pre-cooled $70 \%$ ethanol at $4^{\circ} \mathrm{C}$ for $2 \mathrm{~h}$. The fixed cells were then resuspended in $500 \mu \mathrm{l}$ binding buffer, containing $25 \mu \mathrm{l}$ propidium iodide (PI) and $10 \mu \mathrm{RNase} \mathrm{A}$, at $37^{\circ} \mathrm{C}$ in the dark for $30 \mathrm{~min}$, and detected using flow cytometry.

Hoechst staining. A Hoechst staining kit (Beyotime Institute of Biotechnology) was used to observe the apoptotic nuclear morphology of the cells. Briefly, cells in the logarithmic growth phase were seeded onto cell slides in 12-well plates, at a density of $1 \times 10^{5}$ cells per well, and were cultured for $24 \mathrm{~h}$. Then, the supernatant was discarded. The cells were fixed with $0.5 \mathrm{ml}$ fixing solution at room temperature for $20 \mathrm{~min}$ and were then treated with Hoechst staining solution for $5 \mathrm{~min}$. Anti-fluorescence quenching mounting solution (Beyotime
Institute of Biotechnology) was added dropwise onto the slide, which was then covered with the coverslip containing the cells. The cells were visualized and images were captured under a fluorescence microscope (Olympus BX61; Olympus Corporation, Tokyo, Japan).

Detection of apoptosis using an Annexin V-FITC/PI assay. The cells were seeded into T25 cell culture flasks. When they had reached $90 \%$ confluence, the cells were harvested. The cells were resuspended in $500 \mu$ l Binding Buffer, according to the instructions of the apoptosis detection kit (KeyGEN Biotech, Co., Ltd., Nanjing, China). $5 \mu$ l Annexin V-FITC and $5 \mu \mathrm{l}$ PI were immediately added with thorough mixing. Following $15 \mathrm{~min}$ incubation at $37^{\circ} \mathrm{C}$ in the dark, the cells were detected using flow cytometry (FACSCalibur; BD Biosciences).

Wound healing assay. The cells in each group were seeded into 6-well plates for culture. At a confluence of $80-90 \%$, the culture medium was discarded. Subsequently, a scratch was created on the cell layer using a sterile $200 \mu$ l pipette tip. Images of the cells in each group were captured (AE31; Motic Electric) following being washed twice with serum-free medium. After 12 and $24 \mathrm{~h}$ of continuous culture, the migration distances of the cells were measured, and the cell migration rates were calculated using the following formula: Cell migration rate $=(1-$ distance following healing $/$ distance prior to healing) $\mathrm{x} 100 \%$. Each experiment was repeated three times.

Transwell assay. Following harvesting, $200 \mu 1$ cell suspension containing $2 \times 10^{4}$ cells (resuspended in serum-free culture medium) were seeded in upper Transwell chambers (Corning Incorporated Life Sciences, Tewksbury, MA, USA), which were coated with Matrigel (BD Biosciences), while $800 \mu$ l DMEM medium containing 30\% FBS was added to the lower chamber. Following culture for $24 \mathrm{~h}$, the uninvaded cells on the upper side of the microporous membrane were removed using a cotton swab. The remaining cells were fixed with $4 \%$ paraformaldehyde and stained with $0.5 \%$ crystal violet staining solution (Amresco, Solon, $\mathrm{OH}, \mathrm{USA}$ ). The numbers of invaded cells were recorded under an inverted microscope (AE31; Motic Electric) in five randomly-selected fields (magnification, x200), to obtain the average. 
A
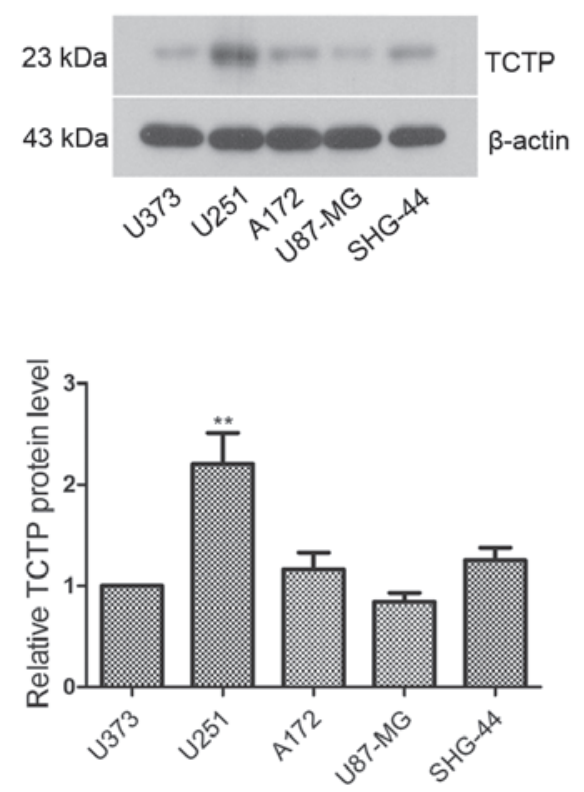

B
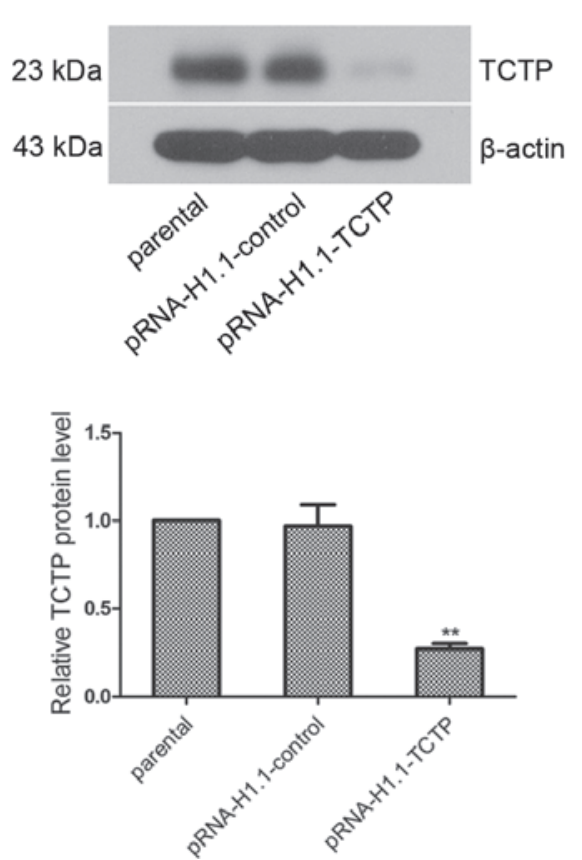

C

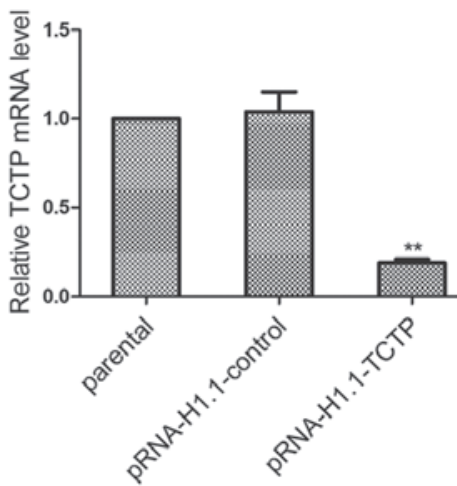

Figure 1. Establishment of a glioma cell line stably transfected with TCTP shRNA. (A) Expression levels of TCTP in the U373, U251, A172, U87-MG and SHG-44 glioma cell lines were compared using western blot analysis, with $\beta$-actin as the internal control. Each experiment was repeated three times. ${ }^{*} \mathrm{P}<0.01$ for U251 cells compared with U373 cells. (B) Protein expressionof TCTP was detected using western blot analysis, with grayscale analysis using $\beta$-actin as an internal control. (C) mRNA expression of TCTP was detected using reverse transcription-quantitative polymerase chain reaction analysis. The data are presented as the mean \pm standard deviations. ${ }^{* *} \mathrm{P}<0.01$, compared with the pRNA-H1.1-control group. TCTP, translationally controlled tumor protein; shRNA, short hairpin ribonucleic acid.

Statistical analysis. The data are presented as the mean \pm standard deviation. One-way analysis of variance was used for comparisons between groups, and Bonferroni's post hoc-test was used for the comparison of multiple variables. The image and data were processed using Graphpad Prism 5.0 software (GraphPad Software, Inc., San Diego, CA, USA). P<0.05 was considered to indicate a statistically significant difference.

\section{Results}

Establishing and identifying the glioma cell line stably transfected with TCTP shRNA. In the present study, total proteins were extracted from the U373, U251, A172, U87-MG and SHG-44 cells to analyze the expression of TCTP in these cells. The results of the western blotting revealed that the expression levels of TCTP in the A172, U87-MG and SHG-44 cells were essentially the same as that in the U373 cells (Fig. 1A; P>0.05), while the level of expression in the U251 cells was significantly higher than that in the U373 cells $(\mathrm{P}<0.01)$. Thus, U251 cells with high expression levels of TCTP were selected to investigate TCTP gene function. The TCTP shRNA eukaryotic expression plasmid, pRNA-H1.1-TCTP, was subsequently transfected into the U251 cells. Western blot and RT-qPCR analyses revealed that the protein and mRNA expression levels of TCTP in the pRNA-H1.1-TCTP group were significantly decreased, and were 0.28 -fold (Fig. 1B; $\mathrm{P}<0.01$ ) and 0.18-fold (Fig. 1C; $\mathrm{P}<0.01)$ lower than that in the pRNA-H1.1-control group, respectively. Thus, a U251 glioma cell line stably transfected with TCTP shRNA was successfully established.
Downregulation of the expression of TCTP suppresses glioma cell proliferation. The impact of the downregulated expression of TCTP on glioma cell proliferation was investigated. The MTT results demonstrated that at 48, 72 and $96 \mathrm{~h}$, cell proliferation in the pRNA-H1.1-TCTP group was significantly lower, compared with that in the pRNA-H1.1-control group (Fig. 2A; P<0.05). The effect of downregulated expression of TCTP on the clonogenic capacity of glioma cells, detected using a colony formation assay, is shown in Fig. 2B. The colony formation rate of the cells in the pRNA-H1.1-TCTP group was significantly lower than that in the pRNA-H1.1-control group (32.0 4 4.9, vs. $60.3 \pm 6.9 \%$, respectively; $\mathrm{P}<0.01)$. The present study further detected the cell cycle distribution using flow cytometry. The percentage of $\mathrm{G} 2 / \mathrm{M}$ phase cells in the pRNA-H1.1-TCTP group was significantly reduced, compared with that in the pRNA-H1.1-control group $(0.56 \pm 0.08$, vs. 8.97 \pm 0.94$) \%$, respectively; Fig. $2 \mathrm{C}$; $\mathrm{P}<0.01)$. The percentage of $\mathrm{S}$ phase cells was essentially unchanged $(26.17 \pm 2.48$, vs. $27.00 \pm 2.23 \%)$, while the percentage of G0/G1 phase cells was significantly increased (73.27 \pm 2.47 , vs. $63.03 \pm 2.11 \%$; $\mathrm{P}<0.01)$. Western blotting revealed that the expression levels of Cyclin D1, Cyclin E and Cyclin B in the pRNA-H1.1-TCTP cells were significantly reduced (Fig. $2 \mathrm{D} ; \mathrm{P}<0.01$ ). These results suggested that downregulation of the expression of TCTP caused cell cycle arrest in the G0/G1 phase and ultimately inhibited glioma cell proliferation.

Downregulation of the expression of TCTP effectively induces the apoptosis of brain glioma cells. The apoptosis 
A

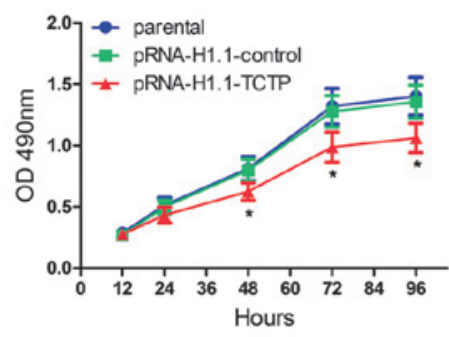

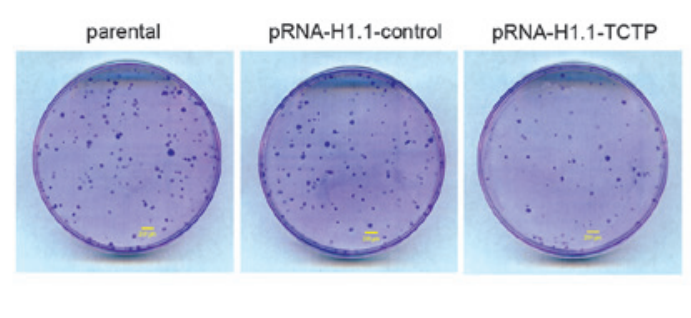

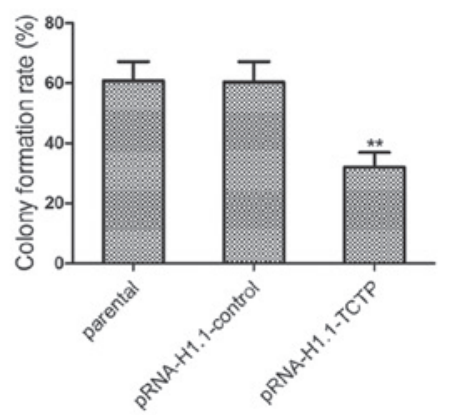

C
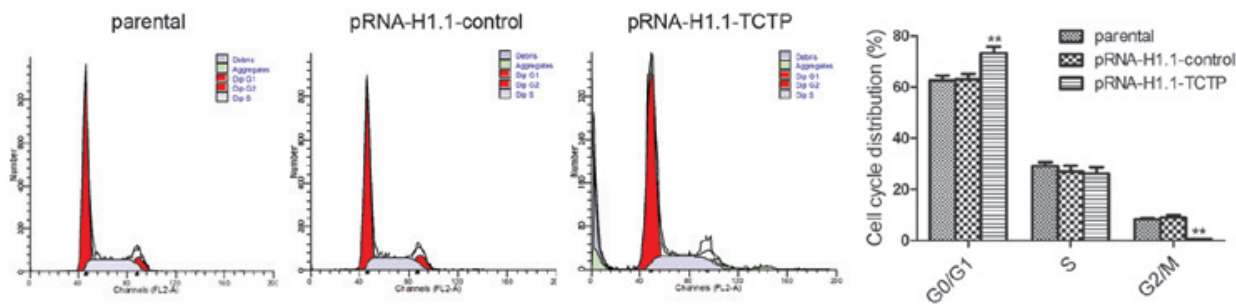

D
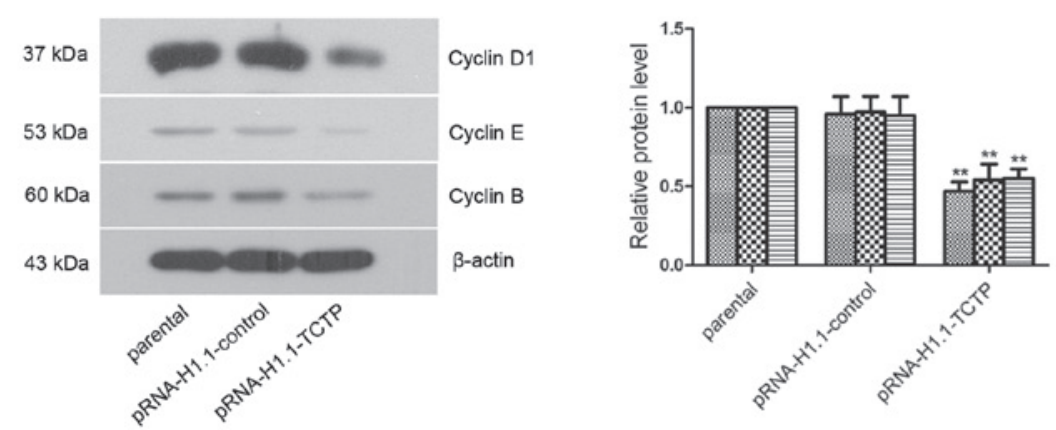

Cyclin D1 Cyclin $E$
Cyclin B

Figure 2. Downregulated expression of TCTP effectively inhibits glioma cell proliferation. (A) Cells of the three groups were seeded in 96-well plates, with five duplicate wells for each setting, and the $\mathrm{OD}_{490}$ values 12, 24, 48, 72 and 96 hafter seeding were detected using an MTT assay. (B) Clonogenic capacity of each group was measured, and a representative image of three repeated experiments are shown (scale bar, $200 \mu \mathrm{m})$. (C) Cell cycle was detected using flow cytometry, and the representative result of three repeated experiments are shown. (D) Expression levels of Cyclin D1, Cyclin E and Cyclin B were detected by western blot analysis. The data are presented as the mean \pm standard deviations. ${ }^{*} \mathrm{P}<0.05$ and ${ }^{* *} \mathrm{P}<0.01$, compared with the pRNA-H1.1-control group. TCTP, translationally controlled tumor protein; shRNA, short hairpin ribonucleic acid; OD, optical density.

and expression levels of apoptosis-associated factors were analyzed using flow cytometry and Hoechst staining. The Hoechst staining revealed that the nuclei of the cells in the parental group and pRNA-H1.1-control group were homogeneous blue. Typical apoptotic morphological changes, including condensed chromatin and shrunken, crumpled and condensed nuclei, were observed in the pRNA-H1.1-TCTP group. The apoptotic rate of the cells in the pRNA-H1.1-TCTP group was significantly higher than that in the pRNA-H1.1-control group (29.6 \pm 3.03 , vs. $6.36 \pm 0.79 \%$, respectively; Fig. $3 \mathrm{~A} ; \mathrm{P}<0.01$ ). Flow cytometry consistently indicated that the apoptotic rate of the cells in the pRNA-H1.1-TCTP group was significantly higher than that in the pRNA-H1.1-control group $(24.60 \pm 2.68$, vs . $6.88 \pm 0.86 \%$, respectively; Fig. $3 \mathrm{~B} ; \mathrm{P}<0.01)$. Western blot analysis demonstrated that the expression levels of Bax and cleaved-caspase-3 in the cells in the pRNA-H1.1-TCTP group were significantly increased (Fig. 3C; $\mathrm{P}<0.01$ ), whereas the expression of $\mathrm{Bcl}-2$ was significantly decreased $(\mathrm{P}<0.01)$. These results suggested that downregulation of the expression of TCTP effectively induced apoptosis in the glioma cells.
Downregulation of the expression of TCTP inhibits the migration and invasion of glioma cells. The present study investigated the impact of downregulated expression of TCTP on glioma cell migration. The cell migration rate of the cells was examined using a wound healing assay, the results of which revealed that the migration rate of the cells in the pRNA-H1.1-TCTP group $12 \mathrm{~h}$ (Fig. 4A; $\mathrm{P}<0.01)$ and $24 \mathrm{~h}(\mathrm{P}<0.05)$ post-wounding were significantly lower than those in the pRNA-H1.1-control group. The effect of TCTP shRNA on the invasiveness of the glioma cells was further detected using a Transwell assay, the results of which revealed that the number of invaded cells in the pRNA-H1.1-TCTP group was significantly lower than that

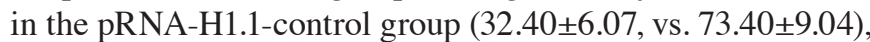
respectively; Fig. 4B; $\mathrm{P}<0.01)$. Western blot analysis revealed that the protein expression levels of MMP-2 and MMP-9 in the cells in the pRNA-H1.1-TCTP group were significantly lower than those in the pRNA-H1.1-control group (Fig. 4C; $\mathrm{P}<0.01$ ). Gelatin zymography revealed bands at 72 and $92 \mathrm{kDa}$ (corresponding to MMP-2 and MMP-9, respectively) in the cells of all groups. Additionally, the activities of MMP-2 and MMP-9 in the cells of the pRNA-H1.1-TCTP group were significantly 
A

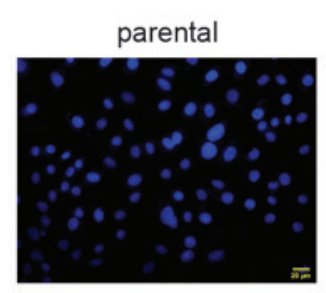

pRNA-H1.1-control
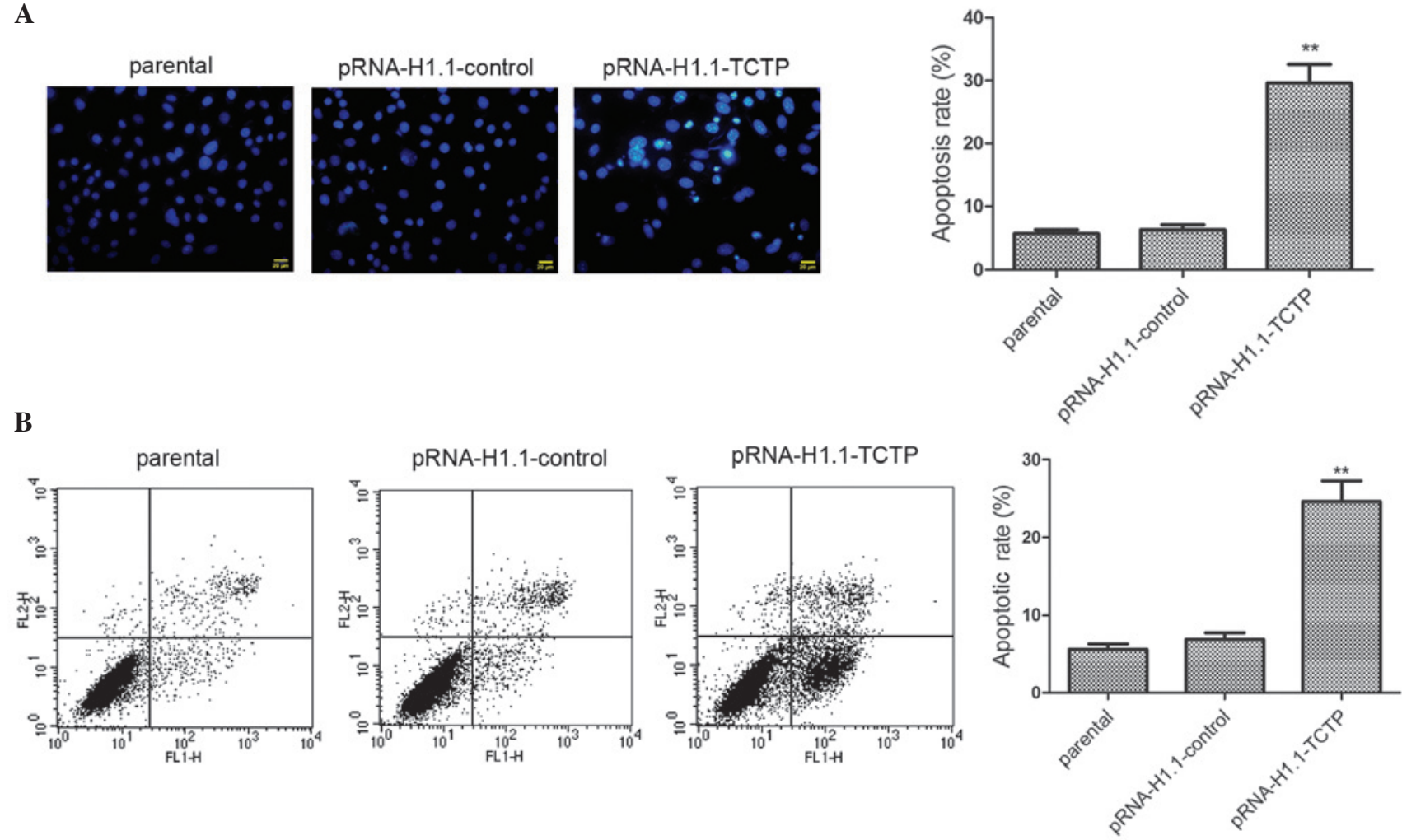

C
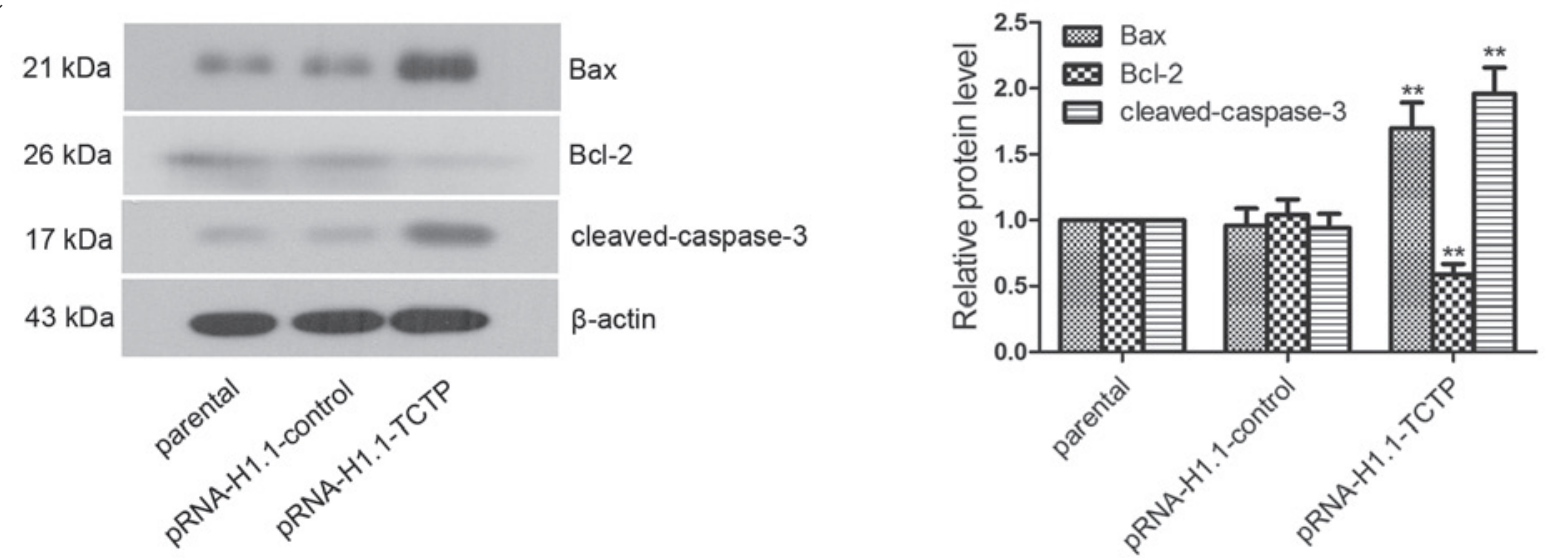

Figure 3. Downregulated expression of TCTP significantly induces apoptosis in glioma cells. (A) Following the downregulation of TCTP, Hoechst staining was performed and apoptosis was observed under a fluorescence microscope. The nuclei of the living cells were homogeneous blue. However, typical apoptotic morphological changes were observed, including condensed chromatin and shrunken, crumpled and condensed nuclei (scale bar, $20 \mu \mathrm{m}$ ). (B) Apoptosis was determined using flow cytometry, and a representative result of the repeated experiments is shown. (C) Expression levels of Bax, Bcl-2, and cleaved-caspase-3 were detected using western blot analysis, with $\beta$-actin as the internal control for grayscale analysis. The data are presented as the mean \pm standard deviations. ${ }^{* *} \mathrm{P}<0.01$, compared with the pRNA-H1.1-control group. TCTP, translationally controlled tumor protein; Bax, B-cell-associated X protein; Bcl-2, B-cell lymphoma-2.

decreased (Fig. 4D; P<0.01). These results demonstrated that downregulation of the expression of TCTP inhibited glioma cell migration and invasion.

\section{Discussion}

TCTP is widely expressed in several types of tumor cell and is important in cell cycle regulation, malignant metastasis and anti-apoptosis (23). However, its role in the malignant metastasis of gliomas remains to be fully elucidated. In the present study, the expression levels of TCTP in various glioma cell lines were detected using western blotting. To investigate the role of TCTP on glioma cell proliferation, cell cycle regulation, apoptosis and invasion, and the associated mechanism, high expression levels of TCTP in the U251 cells was downregulated using shRNA.

An important difference between cancer cells and normal cells is that the growth and division of cancer cells is out of control, and the mechanism of programmed cell death is lacking. The overexpression and downregulation of TCTP can affect tumor cell proliferation $(24,25)$. Studies have reported that hepatoma cell proliferation is significantly inhibited and cell cycle is arrested in the G0/G1 phase following silencing of TCTP using an antisense oligonucleotide (26), and the arrest 
A
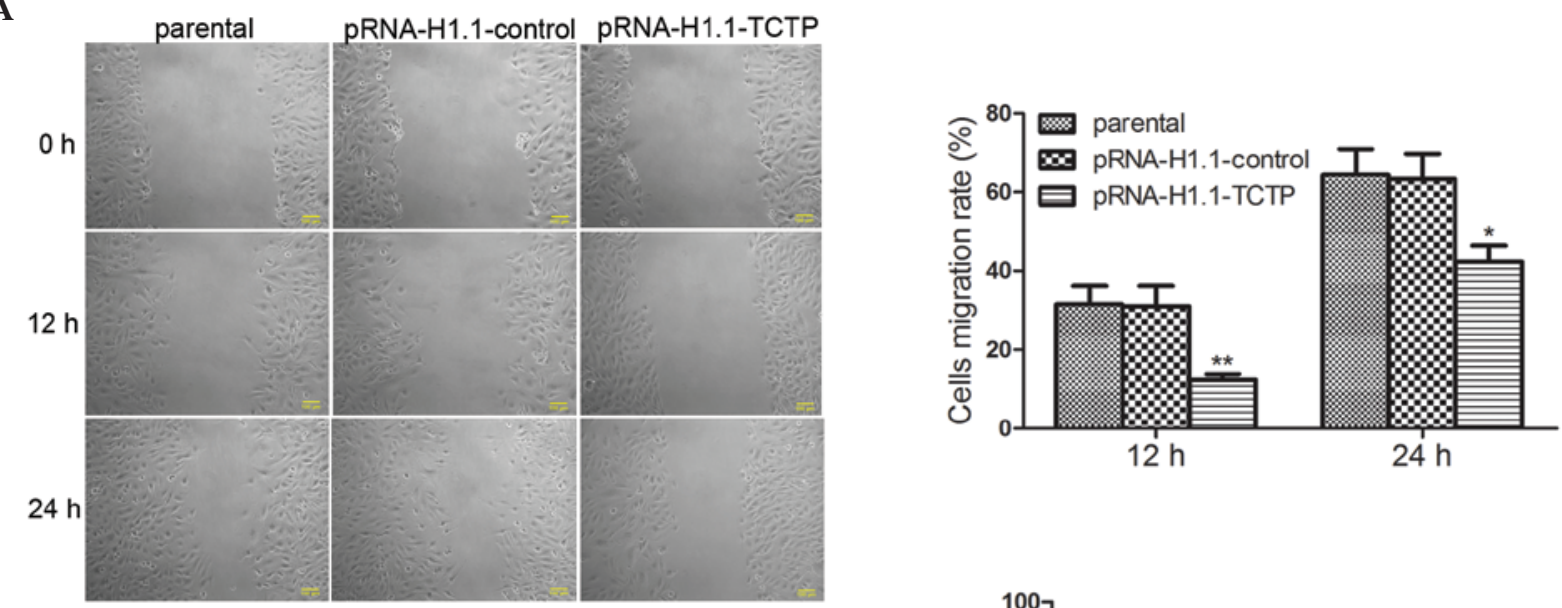

$12 \mathrm{~h}$

$24 \mathrm{~h}$

B

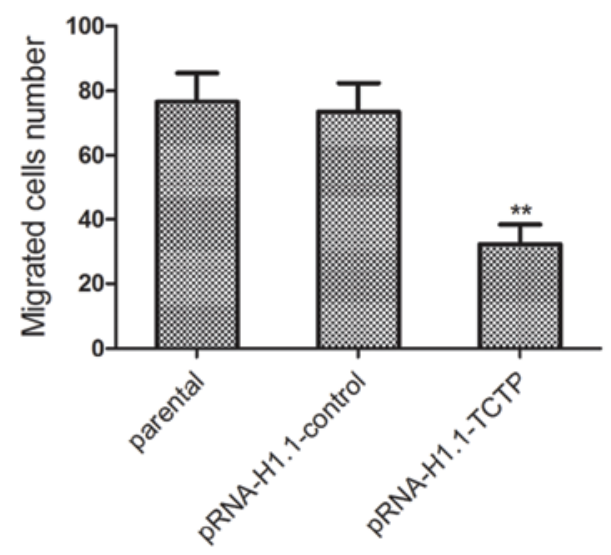

C
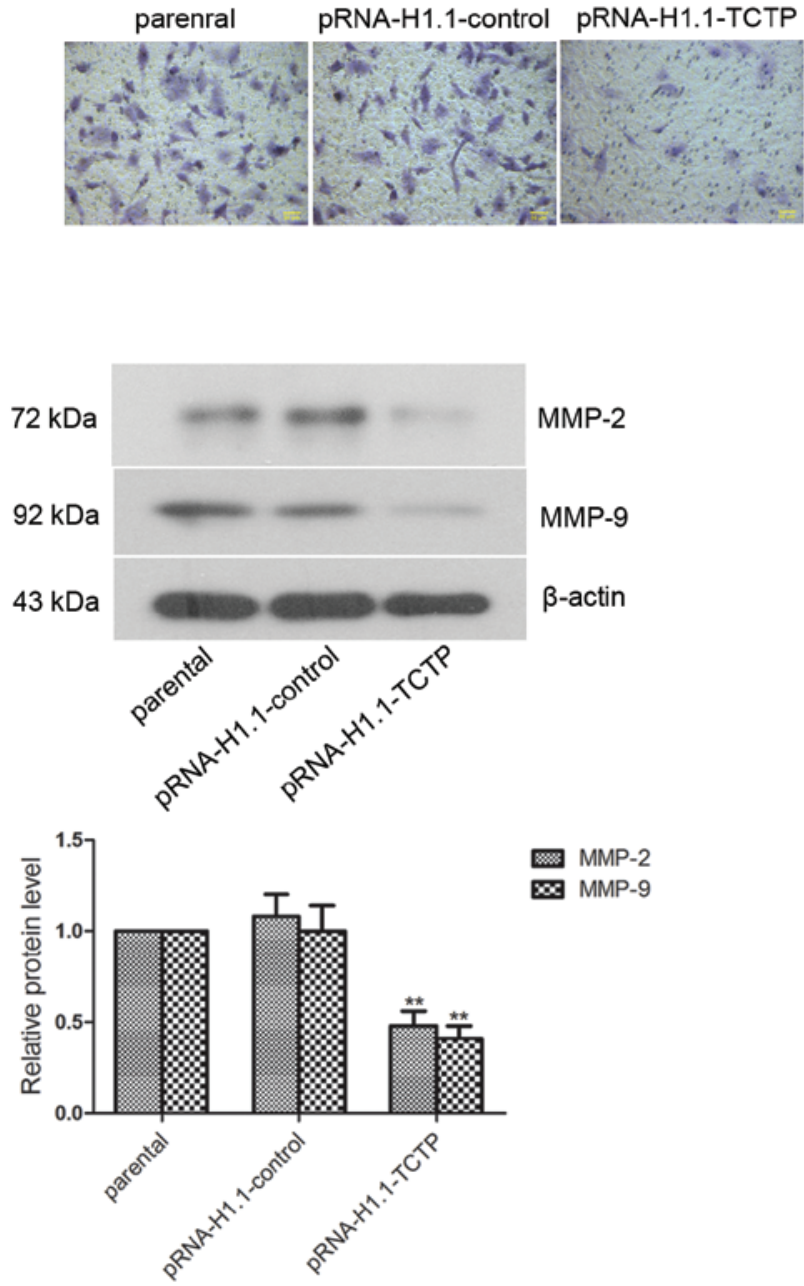

D
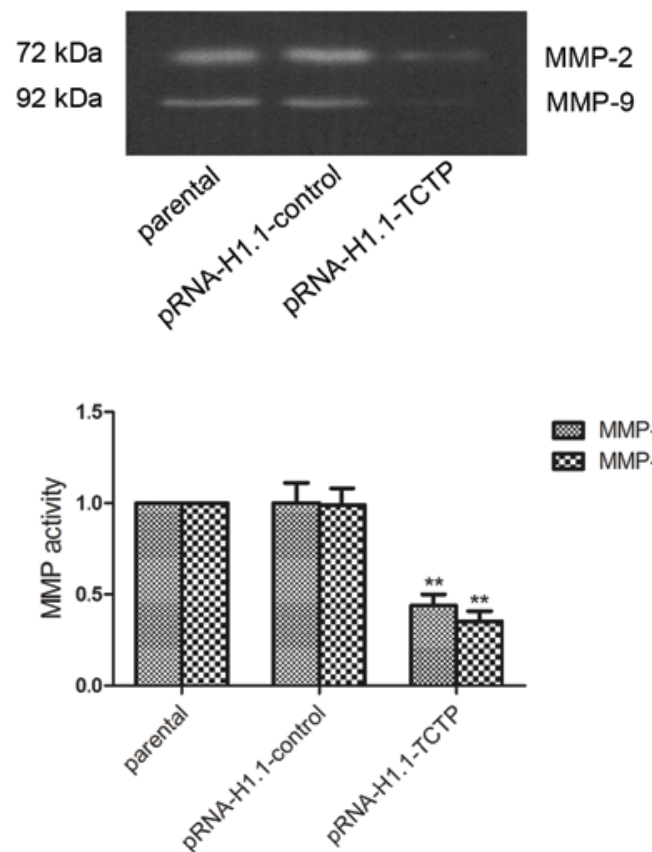

Figure 4. Downregulated expression of TCTP inhibits glioma cell migration and invasion. (A) Cell migration rates of the cells were calculated in each group 24 and $48 \mathrm{~h}$ after scratching in the wound healing assay (scale bar, $100 \mu \mathrm{m}$ ). (B) Invasiveness of the cells was determined using a Transwell assay, and the number of invaded cells was counted under an inverted microscope (scale bar, $50 \mu \mathrm{m}$ ). (C) Protein expression levels of MMP-2 and MMP-9 were detected using western blot analysis. (D) MMP activity levels were assayed using gelatin zymography. The representative result of three repeated experiments is shown. The data are presented as the mean \pm standard deviations. " $\mathrm{P}<0.05$ and ${ }^{* *} \mathrm{P}<0.01$, compared with the pRNA-H1.1-control group. TCTP, translationally controlled tumor protein; shRNA, short hairpin ribonucleic acid; MMP, matrix metalloproteinase.

of the cell cycle is considered to be a predominant reason for the inhibition of tumor cell growth (27-29). The present study investigated the impact of downregulated expression of TCTP on glioma cell proliferation and the cell cycle. The results demonstrated significantly decreased cell proliferation following downregulation of TCTP. Flow cytometric analysis 
determined that the cell cycle was arrested in the G0/G1 phase and that the expression levels of Cyclin D1, Cyclin E and Cyclin B in the cells were significantly lower, compared with those in the control group. This indicated that TCTP regulated the cell cycle by regulating the expression of cell cycle proteins, thereby promoting glioma cell proliferation, which is consistent with the results of previous studies (24-26).

It has been demonstrated that adenovirus vector-driven overexpression of TCTP can effectively resist apoptosis in HeLa cells through the mitochondrial pathway, induced by the chemotherapy drug etoposide, while the activities of cytochrome $c$, caspase-3 and caspase-9 are simultaneously inhibited (30). TCTP can anchor to the mitochondrial membrane to inhibit Bax dimerization, thereby exerting an anti-apoptotic effect through the inhibition of mitochondrial injury (31). Following silencing of the expression of TCTP using a small interfering RNA in breast cancer, squamous cell carcinoma, prostate cancer and lung cancer cells, tumor cell proliferative capability has been observed to decrease, and apoptosis significantly increase (20,32-34). The results of the present study also demonstrated that, following downregulation of the expression of TCTP in glioma cells, the expression levels of Bax and cleaved-caspase- 3 increased, while the expression of $\mathrm{Bcl}-2$ decreased, suggesting that high expression levels of TCTP in glioma cells may exert an anti-apoptotic role by inhibiting Bax and cleaved-caspase-3, and activating $\mathrm{Bcl}-2$, thereby promoting glioma onset and progression.

The metastasis and invasion of tumors is closely associated with their degree of malignancy. MMP-2 and MMP-9 can degrade a variety of extracellular matrices to function in tumor metastasis and invasion (35), which are important in tumor metastasis (36). Previous studies have demonstrated that, following downregulation of the expression of TCTP in colon cancer cells, the abilities of cell proliferation, migration and invasiveness also decrease $(37,38)$. The present study also observed that TCTP is closely associated with glioma cell migration and invasion, as downregulation of TCTP significantly inhibited glioma cell migration and invasion. In addition, the expression and activity levels of MMP-2 and MMP-9 were significantly decreased. These results indicated that TCTP may facilitate glioma cell migration and invasion through MMP-2 and MMP-9.

In conclusion, shRNA-mediated downregulation of the expression of TCTP in brain glioma cells effectively inhibited glioma cell proliferation, promoted apoptosis and inhibited cell migration and invasion. These findings demonstrate the importance of TCTP in the regulation of proliferation, cell cycle, apoptosis and invasion of glioma cells, and offer preliminary evidence for the mechanism underlying its interaction with cell cycle proteins, MMPs and downstream apoptosis-associated factors, and provide a theoretical basis and experimental evidence for using TCTP as a target for gene therapy in glioma.

\section{References}

1. Fine HA, Dear KB, Loeffler JS, Black PM and Canellos GP: Meta-analysis of radiation therapy with and without adjuvant chemotherapy for malignant gliomas in adults. Cancer 71: 2585-2597, 1993.

2. Wu X, Li Y, Wan X, Kayira TM, Cao R, Ju X, Zhu X and Zhao G: Down-regulation of neogenin accelerated glioma progression through promoter Methylation and its overexpression in SHG-44 Induced Apoptosis. PLoS One 7: e38074, 2012.
3. Van Meir EG, Hadjipanayis CG, Norden AD, Shu HK, Wen PY and Olson JJ: Exciting new advances in neuro-oncology: The avenue to a cure for malignant glioma. CA Cancer J Clin 60: 166-193, 2010

4. Turbyville TJ, Gürsel DB, Tuskan RG, Walrath JC, Lipschultz CA, Lockett SJ, Wiemer DF, Beutler JA and Reilly KM: Schweinfurthin A selectively inhibits proliferation and Rho signaling in glioma and neurofibromatosis type 1 tumor cells in a NF1-GRD-dependent manner. Mol Cancer Ther 9: 1234-1243, 2010.

5. Ohgaki H and Kleihues P: Epidemiology and etiology of gliomas Acta Neuropathol 109: 93-108, 2005.

6. Schwartzbaum JA, Fisher JL, Aldape KD and Wrensch M: Epidemiology and molecular pathology of glioma. Nat Clin Pract Neurol 2: 494-503, 2006; quiz 491 p following 516.

7. Gross B, Gaestel M, Böhm H and Bielka H: cDNA sequence coding for a translationally controlled human tumor protein. Nucleic Acids Res 17: 8367, 1989.

8. Chung S, Kim M, Choi W, Chung J and Lee K: Expression of translationally controlled tumor protein mRNA in human colon cancer. Cancer Lett 156: 185-190, 2000.

9. Bazile F, Pascal A, Arnal I, Le Clainche C, Chesnel F and Kubiak JZ: Complex relationship between TCTP, microtubules and actin microfilaments regulates cell shape in normal and cancer cells. Carcinogenesis 30: 555-565, 2009.

10. Zhang F, Liu B, Wang Z, Yu XJ, Ni QX, Yang WT, Mukaida N and Li YY: A novel regulatory mechanism of Pim-3 kinase stability and its involvement in pancreatic cancer progression. Mol Cancer Res 11: 1508-1520, 2013.

11. Liu LK, Wu HF, Guo ZR, Chen XJ, Yang D, Shu YQ and Zhang JN: Targeted efficacy of dihydroartemisinin for translationally controlled protein expression in a lung cancer model. Asian Pac J Cancer Prev 15: 2511-2515, 2014.

12. Cans C, Passer BJ, Shalak V, Nancy-Portebois V, Crible V, Amzallag N, Allanic D, Tufino R, Argentini M, Moras D, et al: Translationally controlled tumor protein acts as a guanine nucleotide dissociation inhibitor on the translation elongation factor eEF1A. Proc Natl Acad Sci USA 100: 13892-13897, 2003.

13. Gachet Y, Tournier S, Lee M, Lazaris-Karatzas A, Poulton T and Bommer UA: The growth-related, translationally controlled protein $\mathrm{P} 23$ has properties of a tubulin binding protein and associates transiently with microtubules during the cell cycle. J Cell Sci 112: 1257-1271, 1999.

14. Liu H, Peng HW, Cheng YS, Yuan HS and Yang-Yen HF: Stabilization and enhancement of the antiapoptotic activity of mcl-1 by TCTP. Mol Cell Biol 25: 3117-3126, 2005.

15. Nagano-Ito M and Ichikawa S: Biological effects of Mammalian translationally controlled tumor protein (TCTP) on cell death, proliferation and tumorigenesis. Biochem Res Int 2012: 204960, 2012.

16. Li F, Zhang D and Fujise K: Characterization of fortilin, a novel antiapoptotic protein. J Biol Chem 276: 47542-47549, 2001.

17. Miao X, Chen YB, Xu SL, Zhao T, Liu JY, Li YR, Wang J, Zhang J and Guo GZ: TCTP overexpression is associated with the development and progression of glioma. Tumour Biol 34: 3357-3361, 2013.

18. Gu X, Yao L, Ma G, Cui L, Li Y, Liang W, Zhao B and Li K: TCTP promotes glioma cell proliferation in vitro and in vivo via enhanced $\beta$-catenin/TCF-4 transcription. Neuro Oncol 16: 217-227, 2014.

19. Acunzo J, Baylot V, So A and Rocchi P: TCTP as therapeutic target in cancers. Cancer Treat Rev 40: 760-769, 2014.

20. Lucibello M, Gambacurta A, Zonfrillo M, Pierimarchi P, Serafino A, Rasi G, Rubartelli A and Garaci E: TCTP is a critical survival factor that protects cancer cells from oxidative stress-induced cell-death. Exp Cell Res 317: 2479-2489, 2011.

21. Graidist P, Phongdara A and Fujise K: Antiapoptotic protein partners fortilin and MCL1 independently protect cells from 5-fluorouracil-induced cytotoxicity. J Biol Chem 279: 40868-40875, 2004.

22. Livak KJ and Schmittgen TD: Analysis of relative gene expression data using real-time quantitative PCR and the 2 (-Delta Delta C(T)) Method. Methods 25: 402-408, 2001.

23. Chan TH, Chen L and Guan XY: Role of translationally controlled tumor protein in cancer progression. Biochem Res Int 2012: 369384, 2012.

24. Tuynder M, Fiucci G, Prieur S, Lespagnol A, Géant A, Beaucourt S, Duflaut D, Besse S, Susini L, Cavarelli J, et al: Translationally controlled tumor protein is a target of tumor reversion. Proc Natl Acad Sci USA 101: 15364-15369, 2004. 
25. Chen SH, Wu PS, Chou CH, Yan YT, Liu H, Weng SY and Yang-Yen HF: A knockout mouse approach reveals that TCTP functions as an essential factor for cell proliferation and survival in a tissue-or cell type-specific manner. Mol Biol Cell 18: 2525-2532, 2007.

26. Zhu WL, Cheng HX, Han N, Liu DL, Zhu WX, Fan BL and Duan FL: Messenger RNA expression of translationally controlled tumor protein (TCTP) in liver regeneration and cancer. Anticancer Res 28: 1575-1580, 2008.

27. Hsiao YC, Hsieh YS, Kuo WH, Chiou HL, Yang SF, Chiang WL and Chu SC: The tumor-growth inhibitory activity of flavanone and 2'-OH flavanone in vitro and in vivo through induction of cell cycle arrest and suppression of cyclins and CDKs. J Biomed Sci 14: 107-119, 2007.

28. Ayyagari VN and Brard L: Bithionol inhibits ovarian cancer cell growth in vitro-studies on mechanism(s) of action. BMC Cancer 14: 61, 2014.

29. He L, Lu N, Dai Q, Zhao Y, Zhao L, Wang H, Li Z, You Q and Guo Q: Wogonin induced G1 cell cycle arrest by regulating $\mathrm{Wnt} / \beta$-catenin signaling pathway and inactivating CDK8 in human colorectal cancer carcinoma cells. Toxicology 312: 36-47, 2013.

30. Jung J, Kim HY, Maeng J, Kim M, Shin DH and Lee K: Interaction of translationally controlled tumor protein with Apaf-1 is involved in the development of chemoresistance in HeLa cells. BMC Cancer 14: 165, 2014.

31. Susini L, Besse S, Duflaut D, Lespagnol A, Beekman C, Fiucci G, Atkinson AR, Busso D, Poussin P, Marine JC, et al: TCTP protects from apoptotic cell death by antagonizing bax function. Cell Death Differ 15: 1211-1220, 2008.
32. Wu D, Guo Z, Min W, Zhou B, Li M, Li W and Luo D: Upregulation of TCTP expression in human skin squamous cell carcinoma increases tumor cell viability through anti-apoptotic action of the protein. Exp Ther Med 3: 437-442, 2012.

33. Rho SB, Lee JH, Park MS, Byun HJ, Kang S, Seo SS, Kim JY and Park SY: Anti-apoptotic protein TCTP controls the stability of the tumor suppressor p53. FEBS Lett 585: 29-35, 2011.

34. Kaarbo M, Storm ML, Qu S, Wæhre H, Risberg B, Danielsen HE and Saatcioglu F: TCTP is an androgen-regulated gene implicated in prostate cancer. PLoS One 8: e69398, 2013.

35. Nakopoulou L, Tsirmpa I, Alexandrou P, Louvrou A, Ampela C, Markaki S and Davaris PS: MMP-2 protein in invasive breast cancer and the impact of MMP-2/TIMP-2 phenotype on overall survival. Breast Cancer Res Treat 77: 145-155, 2003.

36. Luo Y, Liang F and Zhang ZY: PRL1 promotes cell migration and invasion by increasing MMP2 and MMP9 expression through Src and ERK1/2 pathways. Biochemistry 48: 1838-1846, 2009.

37. Chu ZH, Liu L, Zheng CX, Lai W, Li SF, Wu H, Zeng YJ, Zhao HY and Guan YF: Proteomic analysis identifies translationally controlled tumor protein as a mediator of phosphatase of regenerating liver-3-promoted proliferation, migration and invasion in human colon cancer cells. Chin Med J (Engl) 124: 3778-3785, 2011.

38. Ma Q, Geng Y, Xu W, Wu Y, He F, Shu W, Huang M, Du H and $\mathrm{Li} \mathrm{M}$ : The role of translationally controlled tumor protein in tumor growth and metastasis of colon adenocarcinoma cells. J Proteome Res 9: 40-49, 2010. 